\title{
SMART LIGHTING SYSTEM
}

\author{
E.M. DIACONU \\ Valahia University of Targoviste, Faculty of Electrical Engineering, Electronics and Information Technology \\ E-mail: emy_diaconu@yahoo.com
}

\begin{abstract}
This paper proposes a system that allows the control of the lights in a house, building/edifice. The system can be controlled by an application that is made in MIT App Inventor for mobile devices that use Android OS(operating system). The application sends data, via Bluetooth, to the control center, the control center powers on the selected light by turning it on and setting its intensity based on the user preferences. The control center is made from an Arduino Nano programing board, the signal used for powering the lights and setting the brightness is a PWM (Pulse Width Modulation) signal. The system contains the Arduino Nano board, Bluetooth HC-05 module for communication with the mobile application and four LED's that are used to simulate the lights.
\end{abstract}

Keywords: Arduino, Android, Smart lighting system.

\section{INTRODUCTION}

Lately, we have been hearing more and more about "smart lighting", about how smart lighting helps urban agglomerations make the transition to "smart cities" and companies become more efficient. Few of us know, however, what intelligent lighting actually means, how it works and what are the benefits it brings to our homes, the cities we live in or the companies we work for. Smart lighting allows you to remotely control, usually through an application installed on your mobile phone or tablet, lights in a larger or smaller space (city, home, office, etc.). You can control the intensity of the light, the color emitted by the bulbs and, in some cases, you can even put music in the room, if the bulb comes in the package and with a builtin micro-speaker. In other words, you can quickly get the ideal atmosphere for a movie, a romantic dinner, for reading your favorite book or for a restful sleep. Smart lighting uses a system that connects the smart bulbs to a hub connected to a router. It allows other connected devices (such as your mobile phone or tablet) to communicate with and control the bulbs. There are also systems that do not need a hub and can connect directly to Wi-Fi/Bluetooth. It should also be mentioned that intelligent lighting systems cannot be controlled exclusively through applications. Some also allow the use of voice assistants. The Apple HomeKit, the Echo from Amazon or the Home from Google all have voice assistants that can be used to control even the smart bulbs in a building. Smart lighting also cancels the notion of the "switch": when you enter a room, the sensors will detect your presence and turn on the light automatically, without you having to do anything else. We also talk about mobile phones and tablets, becoming intelligent wireless switches that remotely fulfill the role of switch. There are also smart switches similar to the classic ones, but this time they are magnetic and battery-based. They are called "variators" and can be placed anywhere, with a simple adhesive tape. A single dimmer can control up to ten bulbs at a time, with a range of over 12 meters. The system (e.g Figure 1) presented in this paper is made out of three major components:

- Arduino Nano that is used as the light system control.

- Bluetooth HC-05 module

- Four LEDs for the lighting posts.
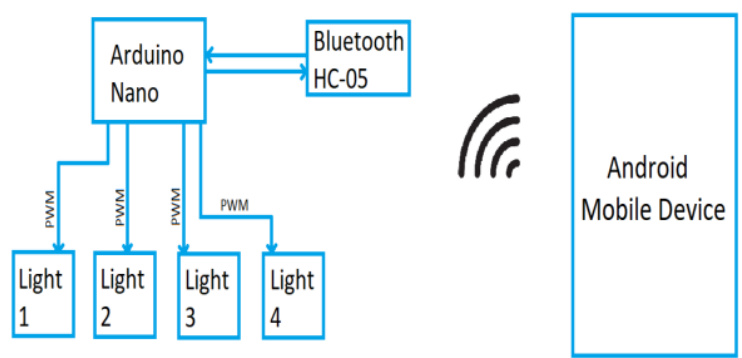

Figure 1. Block diagram.

\subsection{Arduino Nano}

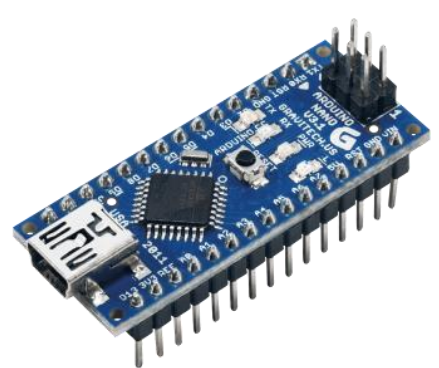

Figure 2. Arduino Nano board.

Arduino Nano (e.g Figure 2) is a small development board $(18 \times 45 \mathrm{~mm})$ and has the technical characteristics similar to the Arduino Uno development board, the only differences being the size and the USB programming / power port. The processor is Atmega328p with a Clock speed of $16 \mathrm{MHz}$ The Arduino Nano development board supports a supply voltage between $5 / 7 \mathrm{~V}-12 \mathrm{~V}$ and has 6 analog pins / 14 digital pins of which 6 pins support PWM (pulse width modulation). The EEPROM memory is $1 \mathrm{kB}$ in size and the SRAM memory is $2 \mathrm{kB}$. The Arduino Nano has a FLASH memory of $32 \mathrm{kB}$ and the communication with the Nano board can be done using various sources, such as using an additional Arduino board, a computer or otherwise using other microcontrollers. The 
microcontroller that is used by the Nano board (ATmega328) provides serial communications (UART TTL). This can be accessed on the digital pins such as TX and RX. The Arduino software includes a serial monitor that allows the transmission and reception of information from the board with easy textual information. Programming an Arduino Nano can be done using Arduino IDE software. The ATmega328 microcontroller on the Nano comes pre-programmed with a bootloader. This bootloader allows you to load new code without using an external hardware programmer. This can be done with the STK500 protocol., here the bootloader can be avoided and the microcontroller program can be achieved using the serial programming header on the circuit or ICSP (In-Circuit -Serial-Programming) with an Arduino ISP(In-System-Programmer).

\subsection{Bluetooth HC-05}

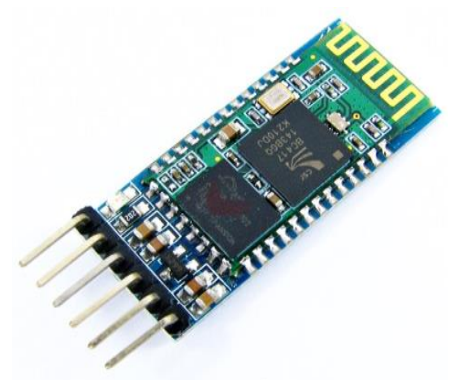

Figure 3. Bluetooth HC-05.

HC-05 (e.g Figure 3) is an easy-to-use module in Arduino projects. Its default configuration is in Slave mode, named HC-05, and the serial interface settings are compatible with the Arduino's default settings (Baud Rate 9600, no parity, 8 data bits, 1 stop bit). The module can be found in the 4-pin version (VCC, GND, RX and TX), or in the 6pin version, which allows the configuration and verification of the status through the program.

Technical specifications:

- Supply voltage: $3.6 \mathrm{~V}-6 \mathrm{~V}$

- Consumption: 30mA

- Coverage radius: max. $100 \mathrm{~m}$

- Uses the standardized IEEE 802.15.1 protocol

- It can be operated as both Master and Slave

- $\quad$ Baud Rate: 9600, 19200, 38400, 57600, 115200, 230400, 460800

Bluetooth is a standardized protocol for data communication using the $2.4 \mathrm{GHz}$ radio frequency. The $2.4 \mathrm{GHz}$ band does not require a license to operate, and is also used by other wireless communication protocols, such as ZigBee or Wi-Fi. As more and more peripherals become wireless, we are never far from the Bluetooth technology we can now find in phones, laptops, headphones, portable speakers, video game controllers, and more.

\subsection{LED}

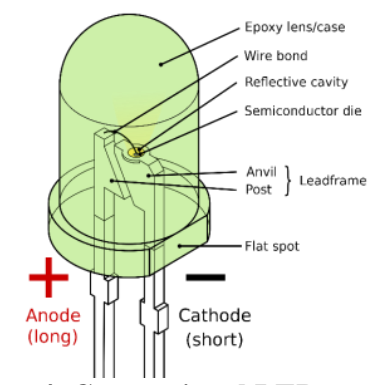

Figure 4. Conventional LED parts.

Light emitting diode (e.g Figure 4) is very commonly known as LED. The light emitting diode (LED) is a semiconductor diode which emits light at the direct polarization of the $\mathrm{p}-\mathrm{n}$ junction. The effect is a form of electroluminescence. An LED is a light source on a small area (less than $1 \mathrm{~mm}$ square) often with an optical system added to the chip to shape the radiation and aid in reflection. The color of the emitted light depends on the composition and conditions of the semiconductor material used, and can be infrared, visible or ultraviolet. Otherwise, in addition to lighting, interesting applications include the use of UV LEDs to sterilize water, disinfect devices and as a larger light source to enhance plant photosynthesis.

\section{SMART LIGHTING SYSTEM}

In Figure 5 we can see the schematic of the PCB for the smart lighting system board that is made from Arduino headers, Bluetooth header, led connectors and the schematic for the lights assembly and in Figure 6 and Figure 7 we have the PCBs.

In Figure 5 we have the schematic of the PCB for the smart lighting system control board and the schematic for the lights assembly.

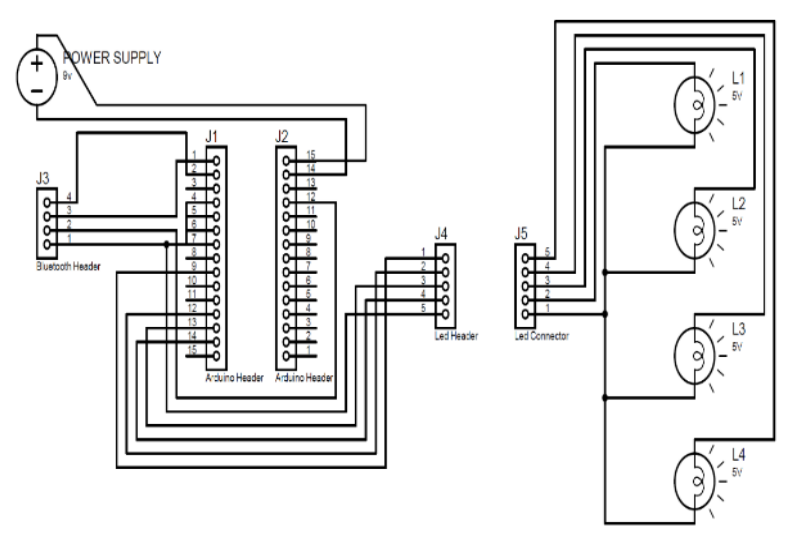

Figure 5. PCB schematic.

In Figure 6 we have the control board that is used for controlling the LED assembly. The control board is made from: power connector (1) that is used to supply power to the Arduino Nano developing board, components and LEDs, Arduino Nano connection headers $(3,4)$ that are used to securely connect the developing board, Bluetooth module header (2) that is used to connect the HC-05 
module and the LED connection pins (5) that are used to supply power and control the LEDs.

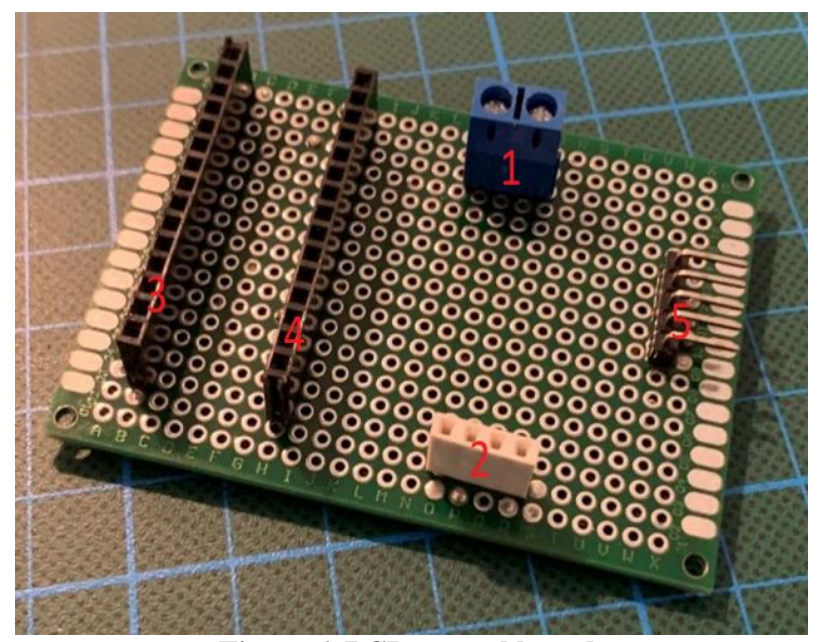

Figure 6. PCB control board.

In Figure 7 we have the LED assembly; the assembly is made from a power and control connector (1) and 4 LEDs $(2,3,4,5)$.

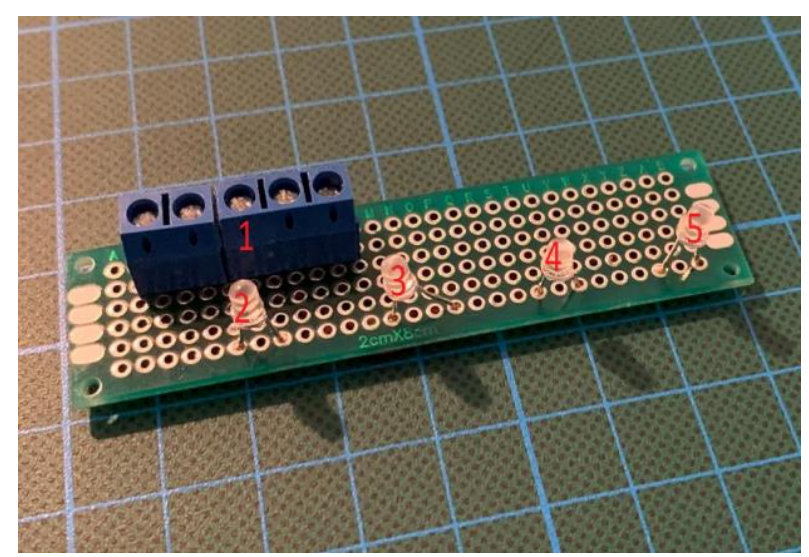

Figure 7. LED assembly.

\section{THE FUNCTIONING PRINCIPLE}

The system has the ability to control 4 LEDs via a Bluetooth connection through an application created in MIT App Inventor. The application is made for the Android OS (operating system) and can be installed on a smartphone or a tablet that uses Android. The control of each LED is made through a PWM signal. The user has the ability to control the brightness besides the on/off function. In Figure 8 we have the smart lighting system fully assembled.

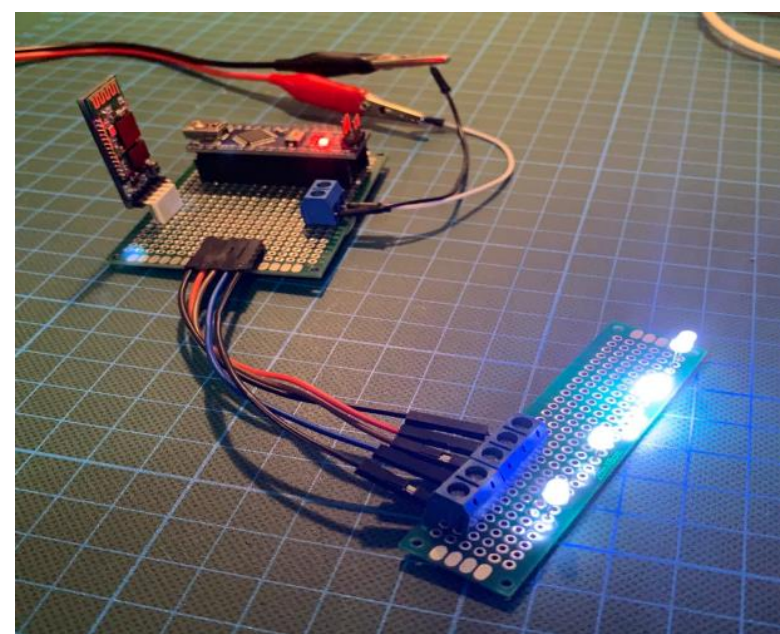

Figure 8. Smart lighting system.

\subsection{Software application}

The control interface (e.g Figure 10) is made from 4 sliders, each LED has a slider. The slider count is from 0 255 exactly as the PWM scale. The user can select a value from 0 to 255 for one of the LEDs, the application sends the value to the control board and then the board gives the LED the PWM output selected from the slider.

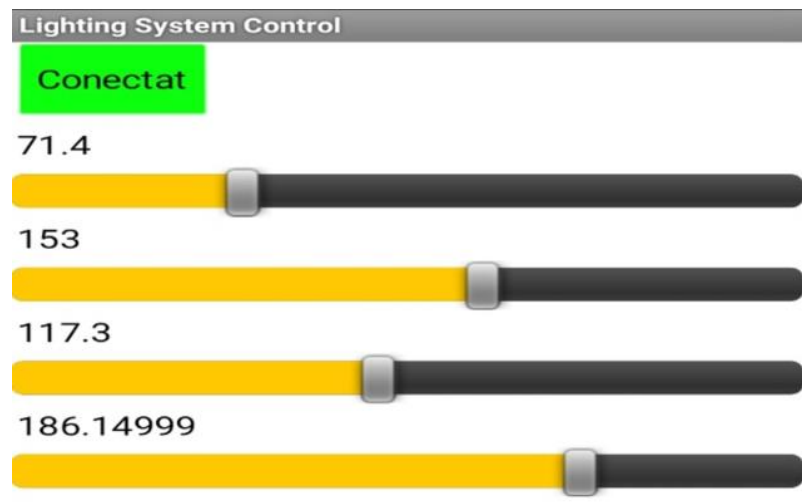

Figure 10. User web interface.

\section{CONCLUSIONS}

A smart lighting system can generate significant energy savings and cost savings by up to $90 \%$, experts say. Sure, this percentage is based on many variables, but, in large part, the figures and examples from real life show that this is possible.

Smart lighting systems can be customized according to the wishes or needs of the user and are more eco-friendly than the classic ones.

Smart lighting system allows people to control every aspect of the lighting system in the home or building from a smart mobile device at 100 meters. Everything becomes much easier to control and manage. 
The system can be implemented in any existing electrical installation by adding power transistors that can be controlled by PWM.

\section{REFERENCES}

[1] Simon Monk, Programming Arduino: Getting Started with Sketches, Second Edition, McGraw Hill Professional, 2016.

[2] Paul Horowitz, Winfield Hill, The Art of Electronics, Cambridge Univeristy Press, 1980.

[3] Herbert Schildt, Java: A Beginner's Guide, Eighth Edition McGraw Hill Professional, 2018.

[4] Cay S. Horstmann, Core Java: Fundamentals. Volume 1, Prentice Hall, 2015.

[5] Jeremy Blum, Exploring Arduino: Tools and Techniques for Engineering Wizardry, John Wiley \& Sons, 2013.

[6] Cuno Pfister, Getting Started with the Internet of Things: Connecting Sensors and Microcontrollers to the Cloud, Maker Media Inc., 2011.

[7] Kevin Townsend, Getting Started with Bluetooth Low Energy: Tools and Techniques for Low-Power Networking, O'Reilly Media Inc., 2014. 